\title{
Unsupervised Domain Clusters in Pretrained Language Models
}

\author{
Roee Aharoni ${ }^{1}$ \& Yoav Goldberg ${ }^{1,2}$ \\ ${ }^{1}$ Computer Science Department, Bar Ilan University \\ ${ }^{2}$ Allen Institute for Artificial Intelligence \\ first.last@gmail.com
}

\begin{abstract}
The notion of "in-domain data" in NLP is often over-simplistic and vague, as textual data varies in many nuanced linguistic aspects such as topic, style or level of formality. In addition, domain labels are many times unavailable, making it challenging to build domainspecific systems. We show that massive pretrained language models implicitly learn sentence representations that cluster by domains without supervision - suggesting a simple datadriven definition of domains in textual data. We harness this property and propose domain data selection methods based on such models, which require only a small set of in-domain monolingual data. We evaluate our data selection methods for neural machine translation across five diverse domains, where they outperform an established approach as measured by both BLEU and by precision and recall of sentence selection with respect to an oracle.
\end{abstract}

\section{Introduction}

It is common knowledge in modern NLP that using large amounts of high-quality training data is a key aspect in building successful machine-learning based systems. For this reason, a major challenge when building such systems is obtaining data in the domain of interest. But what defines a domain? Natural language varies greatly across topics, styles, levels of formality, genres and many other linguistic nuances (van der Wees et al., 2015; van der Wees, 2017; Niu et al., 2017). This overwhelming diversity of language makes it hard to find the right data for the task, as it is nearly impossible to well-define the exact requirements from such data with respect to all the aforementioned aspects. On top of that, domain labels are usually unavailable - e.g. in large-scale web-crawled data like Common Crawl ${ }^{1}$ which was recently used to

\footnotetext{
${ }^{1}$ https://commoncrawl.org/
}

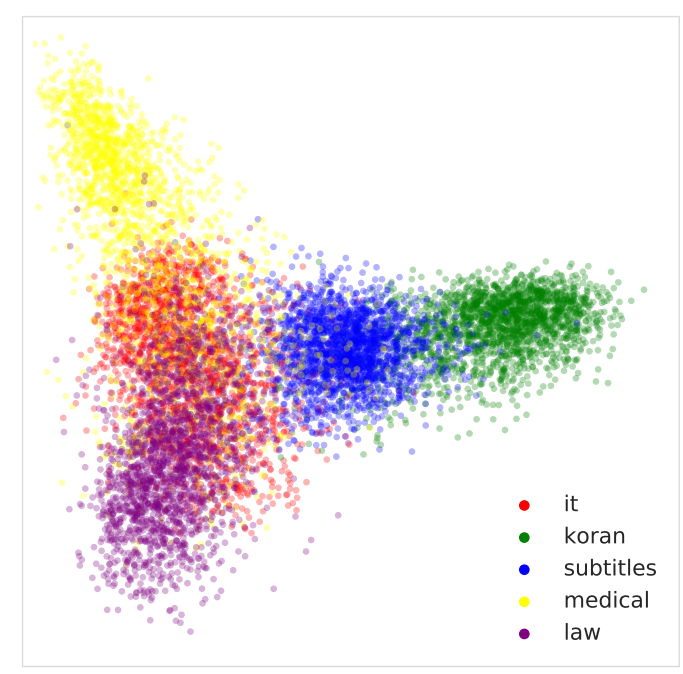

Figure 1: A 2D visualization of average-pooled BERT hidden-state sentence representations using PCA. The colors represent the domain for each sentence.

train state-of-the-art pretrained language models for various tasks (Raffel et al., 2019).

Domain data selection is the task of selecting the most appropriate data for a domain from a large corpus given a smaller set of in-domain data (Moore and Lewis, 2010; Axelrod et al., 2011; Duh et al., 2013; Silva et al., 2018). In this work, we propose to use the recent, highly successful self-supervised pre-trained language models, e.g. Devlin et al. (2019); Liu et al. (2019) for domain data selection. As pretrained LMs demonstrate state-of-theart performance across many NLP tasks after being trained on massive amounts of data, we hypothesize that the robust representations they learn can be useful for mapping sentences to domains in an unsupervised, data-driven approach. We show that these models indeed learn to cluster sentence representations to domains without further supervision (e.g. Figure 1), and quantify this phenomenon by fitting Gaussian Mixture Models (GMMs) to the learned representations and measuring the purity of the resulting unsupervised clustering. We then pro- 
pose methods to leverage these emergent domain clusters for domain data selection in two ways:

- Via distance-based retrieval in the sentence embedding space induced by the pretrained language model.

- By fine-tuning the pretrained language model for binary classification, where positive examples are from the domain of interest.

Our methods enable to select relevant data for the task while requiring only a small set of monolingual in-domain data. As they are based solely on the representations learned by self-supervised LMs, they do not require additional domain labels which are usually vague and over-simplify the notion of domain in textual data. We evaluate our method on data selection for neural machine translation (NMT) using the multi-domain GermanEnglish parallel corpus composed by Koehn and Knowles (2017). Our data selection methods enable to train NMT models that outperform those trained using the well-established cross-entropy difference method of Moore and Lewis (2010) across five diverse domains, achieving a recall of more than $95 \%$ in all cases with respect to an oracle that selects the "true" in-domain data.

Our contributions in this work are as follows. First, we show that pre-trained language models are highly capable of clustering textual data to domains with high accuracy in a purely unsupervised manner. Second, we propose methods to select in-domain data based on this property using vectorspace retrieval and positive-unlabeled fine-tuning of pretrained language models for binary classification. Third, we show the applicability of our proposed data selection methods on a popular benchmark for domain adaptation in machine translation. An additional contribution is a new, improved data split we create for this benchmark, as we point on issues with previous splits used in the literature. The code and data for this work is publicly available. ${ }^{2}$ We hope this work will encourage more research on understanding the data landscape in NLP, enabling to "find the right data for the task" in the age of massive models and diverse data sources.

\footnotetext{
${ }^{2}$ https://github.com/roeeaharoni/ unsupervised-domain-clusters
}

\section{Emerging Domain Clusters in Pretrained Language Models}

\subsection{Motivation}

The proliferation of massive pretrained neural language models such as ELMo (Peters et al., 2018), BERT (Devlin et al., 2019) or RoBERTa (Liu et al., 2019) has enabled great progress on many NLP benchmarks (Wang et al., 2018, 2019a). Larger and larger models trained on billions of tokens of raw text are released in an ever-increasing pace (Raffel et al., 2019), enabling the NLP community to fine-tune them for the task of interest. While many works tried to "probe" those models for the morphological, syntactic and semantic information they capture (Tenney et al., 2019; Goldberg, 2019; Clark et al., 2019), an important aspect of language remained overlooked in this context - the domain the data comes from, often referred to as the "data distribution".

The definition of domain is many times vague and over-simplistic (e.g. "medical text" may be used for biomedical research papers and for clinical conversations between doctors and patients, although the two vary greatly in topic, formality etc.). A common definition treats a domain as a data source: "a domain is defined by a corpus from a specific source, and may differ from other domains in topic, genre, style, level of formality, etc." (Koehn and Knowles, 2017). We claim that a more data-driven definition should take place, as different data sources may have sentences with similar traits and vice versa - a single massive web-crawled corpus contains texts in numerous styles, topics and registers. Our analysis in Section 2 shows examples for such cases, e.g. a sentence discussing "Viruses and virus-like organisms" in a legal corpus.

We hypothesize that massive pretrained LMs can learn representations that cluster to domains, as texts from similar domains will appear in similar contexts. We test this hypothesis across several large, publicly-available pretrained LMs; we explore both masked-language-models (MLMs) and auto-regressive LMs.

\subsection{Method}

We encode multi-domain data at the sentence level into vector representations. We then cluster these vector representations for each model using a Gaussian Mixture Model (GMM) with $k$ pre-defined clusters. We chose GMM as our clustering approach as it allows soft assignments (vs. hard as- 


\begin{tabular}{|l|c|c|c|}
\cline { 2 - 4 } \multicolumn{1}{c|}{} & $\mathrm{k}=5$ & $\mathrm{k}=10$ & $\mathrm{k}=15$ \\
\hline Random & $15.08( \pm 0.0)$ & $16.77( \pm 0.0)$ & $17.78( \pm 0.0)$ \\
\hline LDA & $24.31( \pm 0.99)$ & $26.73( \pm 2.19)$ & $30.79( \pm 2.97)$ \\
\hline
\end{tabular}

\begin{tabular}{|l|c|c|c|c|c|c|}
\multicolumn{1}{c}{} & \multicolumn{3}{c}{ with PCA $(\mathrm{n}=50)$} & \multicolumn{3}{c|}{ without PCA } \\
\cline { 2 - 7 } \multicolumn{1}{c|}{} & $\mathrm{k}=5$ & $\mathrm{k}=10$ & $\mathrm{k}=15$ & $\mathrm{k}=5$ & $\mathrm{k}=10$ & $\mathrm{k}=15$ \\
\hline word2vec & $53.65( \pm 0.79)$ & $68.14( \pm 2.58)$ & $73.44( \pm 0.68)$ & 45.93 & 65.80 & 76.26 \\
\hline BERT-base & $\mathbf{8 7 . 6 6}( \pm \mathbf{0 . 2 4})$ & $88.02( \pm 1.10)$ & $88.37( \pm 0.66)$ & $\mathbf{8 5 . 7 4}$ & 85.08 & 86.37 \\
\hline BERT-large & $85.64( \pm 6.13)$ & $87.61( \pm 0.26)$ & $89.07( \pm 0.53)$ & 68.56 & $\mathbf{8 6 . 5 3}$ & 86.99 \\
\hline DistillBERT & $83.68( \pm 7.14)$ & $86.31( \pm 0.86)$ & $87.53( \pm 0.85)$ & 79.00 & 86.42 & $\mathbf{8 8 . 1 4}$ \\
\hline RoBERTa-base & $79.05( \pm 0.10)$ & $86.39( \pm 0.90)$ & $86.51( \pm 0.28)$ & 70.21 & 80.35 & 81.49 \\
\hline RoBERTa-large & $80.61( \pm 0.33)$ & $\mathbf{8 9 . 0 4}( \pm \mathbf{0 . 1 5})$ & $\mathbf{8 9 . 9 4}( \pm \mathbf{0 . 2 3})$ & 69.88 & 81.07 & 85.91 \\
\hline GPT-2 & $70.30( \pm 0.05)$ & $84.76( \pm 0.30)$ & $82.56( \pm 1.29)$ & 37.82 & 39.02 & 41.45 \\
\hline XLNet & $55.72( \pm 0.69)$ & $68.17( \pm 3.93)$ & $72.65( \pm 1.92)$ & 30.36 & 32.96 & 48.55 \\
\hline
\end{tabular}

Table 1: Unsupervised domain clustering as measured by purity for the different models. Best results are marked in bold for each setting.

signments as in e.g. K-means) which we think fits the task better (as a sentence can be seen as drawn from a mixture of several domain). ${ }^{3}$ In all cases, to create a sentence representation we perform average pooling of the last hidden state (before the softmax layer) for each token in the sentence. ${ }^{4}$ To accelerate the clustering process and enable visualization we also experiment with performing dimensionality reduction with PCA over the sentence vectors before clustering them. We experiment with $\mathrm{k}$ in 5, 10 and 15 to test how adding flexibility would improve the domain clustering accuracy.

\subsection{Models and Baselines}

For MLM-based models we use BERT (Devlin et al., 2019), DistilBERT (Sanh et al., 2019) and RoBERTa (Liu et al., 2019) (in both the base and large versions). For autoregressive models we use GPT-2 (Radford et al., 2018) and XLNet (Yang et al., 2019). In all cases we use the implementations from the HuggingFace Transformers toolkit (Wolf et al., 2019). We also evaluated three additional, simpler baselines. The first is using representations from word2vec (Mikolov et al., 2013), where we average-pooled the word vectors for the tokens that were present in the model vocabulary. The second is using Latent Dirichlet Allocation (LDA, Blei et al., 2003), which is a classic approach to unsupervised clustering of text. ${ }^{5} \mathrm{We}$ also

\footnotetext{
${ }^{3}$ See further discussion comparing GMMs and K-means in Daume (2009).

${ }^{4}$ Using the penultimate layer or others may result in better performance; we leave this for future work.

${ }^{5}$ We used the LDA implementation provided in the Gensim toolkit: https: //radimrehurek.com/gensim/
}

report results for a baseline which assigns sentences by sampling randomly from a uniform distribution over the clusters.

\subsection{Evaluation}

To evaluate the unsupervised domain clustering we used the multi-domain corpus proposed by Koehn and Knowles (2017) which includes textual data in five diverse domains: subtitles ${ }^{6}$, medical text (PDF documents from the European Medicines Agency), legal text (legislative text of the European Union), translations of the Koran, and IT-related text (manuals and localization files of open-source software). This dataset includes parallel sentences in English and German; for this experiment we used the English portion of the data. See more details on the dataset in Section 3.1. We used 2000 distinct sentences from each domain. To evaluate whether the resulting clusters indeed capture the domains the data was drawn from we measure the clustering purity, which is a well-known metric for evaluating clustering (Manning et al., 2008). To measure the clustering purity, we assign each unsupervised cluster with the most common "true" domain in the sentences assigned to that cluster, and then compute the accuracy according to this majority-based cluster-domain assignment (note that in this case several unsupervised clusters can be assigned to the same domain). In cases where randomness is involved we run each experiment five times with different initializations and report the mean and variance of the purity metric for each model.

\footnotetext{
${ }^{6}$ From http: / / www. opensubtitles.org/
} 


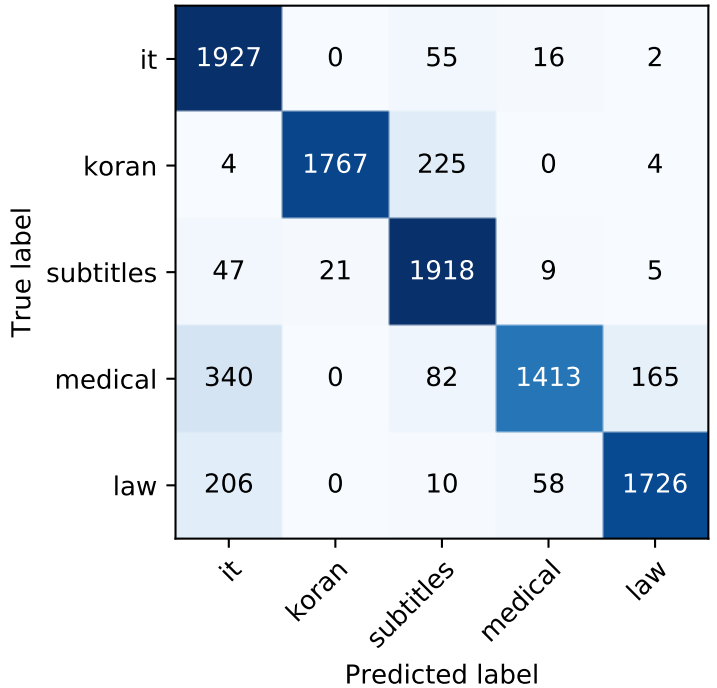

Figure 2: A confusion matrix for clustering with $\mathrm{k}=5$ using BERT-base.

\subsection{Results and Discussion}

As can be seen in Table 1, pre-trained language models are indeed highly capable of generating sentence representations that cluster by domains, resulting in up to $87.66 \%, 89.04 \%$ and $89.94 \%$ accuracy when using $\mathrm{k}=5, \mathrm{k}=10$ and $\mathrm{k}=15$ clusters, respectively, across 10,000 sentences in 5 domains. We find these scores remarkably high given our straight-forward average-pooling strategy and that no domain-supervision was involved in the process of learning the pre-trained representations. Figure 3 also demonstrates the quality of the obtained clusters in 2D using the BERT-base model, where the ellipses describe the mean and variance parameters learned for each cluster by the GMM with $k=5 .^{7}$

We note that some classes of models did better than others: while all vector-based models did far better than the random and LDA baselines ${ }^{8}$, the MLM-based models dominated in all cases over word2vec and the auto-regressive models. This may be explained by the fact that the MLM-based models use the entire sentence context when generating the representations for each token, while the auto-regressive models only use the past context, and word $2 \mathrm{vec}$ uses a limited window context. Using PCA improved performance in most cases and especially for the auto-regressive models, although the results for the MLMs remain high in

\footnotetext{
${ }^{7}$ Similar visualizations for additional models are available in the supplementary material.

${ }^{8}$ Note that the LDA models were trained using the multidomain data alone, and did not utilize additional pretraining as in the other, more successful models. This may explain their relatively weak performance.
}

both cases - suggesting that these models encode the information very differently.

\subsection{Analysis}

As can be seen in Figure 3, in some areas the domains are somewhat overlapping in the embedding space, which may lead to outlier cases where examples from one domain are assigned to a cluster of a another domain. We plot a confusion matrix (Figure 2) to analyze this further based on the clustering with BERT-base and $\mathrm{k}=5$. We first note that the outlier sentences are much shorter than the average sentence length in the corpus (11.62 tokens on average for outliers vs. 20.5 tokens on average in general). This makes sense as shorter sentences contain less information, making it harder to assign them to an appropriate cluster. Table 2 shows examples of outlier sentences, assigned to clusters of domains different from their originating domain. We can see that in many cases the assignments are sensible - for example for sentences originating from the subtitles corpus, a sentence that mentions "great priest" is assigned to the Koran cluster, a sentence that mentions "The International Criminal Court in The Hague" is assigned to the Law cluster, a sentence that mentions "the virus" is assigned to the Medical cluster and so on. This strengthens our claim that defining domains based on the corpus they originated from is over-simplistic, and using a data-driven approach may enable to find better domain assignments across different corpora.

The domain that attracted the largest number of outliers is the IT domain cluster, with 597 sentences assigned to it from other domains. Looking

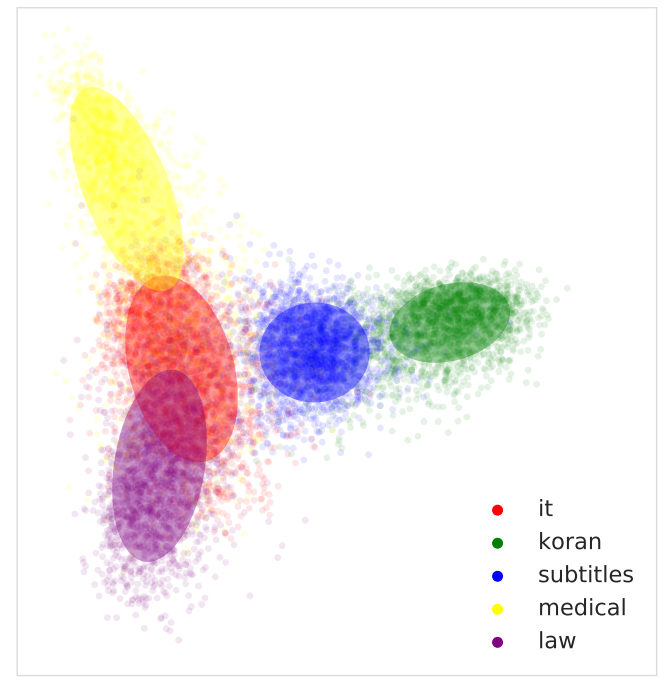

Figure 3: A 2D visualization of the unsupervised GMM clustering for the same sentences as in Figure 1. 


\begin{tabular}{|l|l|}
\hline \multicolumn{1}{|c|}{ Subtitles assigned to Koran } & \multicolumn{1}{c|}{ Subtitles assigned to Medical } \\
\hline I am Spa'am, high priest of the boars. & Oxygen supply at 50\%. \\
\hline Joseph, go in peace, and the Lord be with you. & $\begin{array}{l}\text { Or it can help her walk again if the virus is kept in check } \\
\text { with this. }\end{array}$ \\
\hline \multicolumn{1}{|c|}{ Subtitles assigned to IT } & \multicolumn{1}{c|}{ Subtitles assigned to Law } \\
\hline Push it up to the front of the screen. & Statutes, transcripts, redacted immunity agreements. \\
\hline $\begin{array}{l}\text { Polyalloy requires programming to take permanent } \\
\text { form. }\end{array}$ & $\begin{array}{l}\text { The Security Council therefore must press for his immediate } \\
\text { referral to the International Criminal Court in The Hague. }\end{array}$ \\
\hline \multicolumn{1}{|c|}{ Lawed to Medical } & \multicolumn{1}{c|}{ Lawsigned to IT } \\
\hline - Viruses and virus-like organisms & "INFORMATION SOCIETY STATISTICS \\
\hline $\begin{array}{l}\text { where the glucose content is equal to or less than } \\
\text { the fructose content. }\end{array}$ & $\begin{array}{l}\text { This document must be attached to the certificate and field } \\
\text { with it, except where there is a computerised checking system. }\end{array}$ \\
\hline \multicolumn{2}{|c|}{ Medical assigned to IT } \\
\hline $\begin{array}{l}\text { This will be introduced by a Regulation adopted by the } \\
\text { European Commission. }\end{array}$ & $\begin{array}{l}\text { An updated and improved version of the CD-ROM was issued } \\
\text { to all subscribers during the first half of the year. }\end{array}$ \\
\hline $\begin{array}{l}\text { The marketing authorisation was renewed on 22 May } \\
\text { 2002 and 22 May 2007. }\end{array}$ & $\begin{array}{l}\text { - All tables will be based on generic and not product-specific } \\
\text { data. }\end{array}$ \\
\hline \multicolumn{2}{|c|}{ IT assigned to Subtitles } \\
\hline R65: Harmful: may cause lung damage if swallowed & At the end we say good bye. \\
\hline Automatic Red-Eye Removal & What would you like to do for your next shot? \\
\hline \multicolumn{2}{|c}{}
\end{tabular}

Table 2: Sentences from one domain which were assigned to another domain by the BERT-based clustering, $\mathrm{k}=5$.

more closely we find that more than half of these sentences (340 out of 597) included numbers (e.g. "34\% 25\% 34\%" (from medical), "(b) reference number 20 is deleted;" (from law), "(Command of Prostration \# 1)" (from Koran) or "The message, R2." (from subtitles)). As numbers appear in many different contexts, they may be harder to assign to a specific domain by the context-aware language models in such short sentences. The second largest attractor of outliers is the Subtitles cluster, with 372 sentences assigned to it from other domains. We find that most of these sentences contain personal pronouns or question marks (228 out of 372, $61.2 \%$ ) while the ratio of such sentences in the entire corpus is only $40 \%$. Examples include "Why did you choose the name \& amarok;?" (from IT), or "What is Avonex?" (from Medical). This may be expected as the subtitles corpus mainly includes transcriptions of spoken, conversational language, and "conversation tends to have more verbs, more personal pronouns, and more questions" (Conrad and Biber, 2005). Another possible reason for the subtitles domain to attract outliers is the fact that this is the least-topical cluster: movies and TV series may discuss diverse topics, unlike medical, religious, legal and technical texts that may have a more cohesive topic.

\section{Neural Machine Translation in a Multi-Domain Scenario}

As we showed that pre-trained language models are indeed very useful in clustering sentence representations by domains in an unsupervised manner, we now seek to harness this property for a down- stream task - domain data selection for machine translation. Domain data selection is the task of selecting examples from a large corpus which are as close as possible to the domain of interest, given a smaller set of in-domain examples. The selected examples can be used to either (1) train a domainspecific model from scratch (Axelrod et al., 2011), (2) fine-tune a pre-trained general-domain model (Sajjad et al., 2017; Silva et al., 2018), or (3) prioritize data for annotation as in an Active-Learning framework, if only monolingual data is available (Haffari et al., 2009). To demonstrate the need for domain data selection and set the stage for our data selection experiments, we perform preliminary experiments with NMT in a multi-domain scenario.

\subsection{Multi-Domain Dataset}

To simulate a diverse multi-domain setting we use the dataset proposed in Koehn and Knowles (2017), as it was recently adopted for domain adaptation research in NMT (Hu et al., 2019; Müller et al., 2019; Dou et al., 2019a,b). The dataset includes parallel text in German and English from five diverse domains (Medical, Law, Koran, IT, Subtitles; as discussed in Section 2), available via OPUS (Tiedemann, 2012; Aulamo and Tiedemann, 2019).

In a preliminary analysis of the data we found that in both the original train/dev/test split by Koehn and Knowles (2017) and in the more recent split by Müller et al. (2019) there was overlap between the training data and the dev/test data. ${ }^{9}$ Fixing these issues is important, as it may affect the conclusions one draws from experiments with

\footnotetext{
${ }^{9}$ More details are available in the supplementary material.
} 


\begin{tabular}{|c|c|c|}
\cline { 2 - 3 } \multicolumn{1}{c|}{} & Original & New Split \\
\hline Medical & $1,104,752$ & 248,099 \\
\hline Law & 715,372 & 467,309 \\
\hline IT & 378,477 & 222,927 \\
\hline Koran & 533,128 & 17,982 \\
\hline Subtitles & $22,508,639$ & $14,458,058$ \\
\hline
\end{tabular}

Table 3: Number of training examples for each domain in the original split (Müller et al., 2019) and in our split.

this dataset. For example, as overlapping development sets favor memorization of the training set, one may choose checkpoints and report results on over-fitting models. This is especially relevant with neural sequence-to-sequence models, as they are highly susceptible to memorization (Aharoni and Goldberg, 2018) and hallucination (Lee et al., 2018), as confirmed by Müller et al. (2019).

To create a better experimental setting to test generalization within and across domains, we create a new data split where we ensure that no such overlap between the training, development and test sets occur. We started from the split of Müller et al. (2019) as it included newer versions of some of the datasets. ${ }^{10}$ Furthermore, we did not allow more than one translation of a given source or target sentence, as such cases were very frequent in the dataset and usually stand for duplicate sentence pairs (See Table 3). For example, applying this filtering reduced the size of the Koran corpus from 533,128 sentence pairs to only 17,982 . Finally, following Müller et al. (2019) we cap the subtitles corpus to 500,000 sentence pairs as it is much larger than the rest. We make the new split publicly available and hope it will enable better future experimentation on this important subject. ${ }^{11}$

\subsection{Cross-Domain Experiments}

Experimental Setup We follow Hu et al. (2019) and train domain-specific models for all domains. We then evaluate each model across the different domain test sets, enabling us to understand the effect of different domains on the downstream MT performance and to set up strong baselines for data selection experiments. We also train a generaldomain model using the available data from all domains, as it is also a common approach in multidomain scenarios (Müller et al., 2019). In all experiments we use a similar Transformer (Vaswani et al., 2017) model, and only control for the train-

\footnotetext{
${ }^{10}$ Their dataset is available in: https: / / github. com/ ZurichNLP/domain-robustness

${ }^{11}$ https://github.com/roeeaharoni/ unsupervised-domain-clusters
}

\begin{tabular}{|c|c|c|c|c|c|}
\cline { 2 - 6 } \multicolumn{1}{c|}{} & Medical & Law & Koran & IT & Subtitles \\
\hline Medical & $\mathbf{5 6 . 5}$ & 18.3 & 1.9 & 11.4 & 4.3 \\
\hline Law & 21.7 & $\mathbf{5 9}$ & 2.7 & 13.1 & 5.4 \\
\hline Koran & 0.1 & 0.2 & 15.9 & 0.2 & 0.5 \\
\hline IT & 14.9 & 9.6 & 2.8 & $\mathbf{4 3}$ & 8.6 \\
\hline Subtitles & 7.9 & 5.5 & 6.4 & 8.5 & 27.3 \\
\hline All & 53.3 & 57.2 & $\mathbf{2 0 . 9}$ & 42.1 & $\mathbf{2 7 . 6}$ \\
\hline
\end{tabular}

Table 4: SacreBLEU (Post, 2018) scores of our baseline systems on the test sets of the new data split. Each row represents the results from one model on each test set. The best result in each column is marked in bold.

ing data. More details on the exact training and hyperparameter settings for the NMT models are available in the supplementary material.

Results The results for the cross-domain evaluation are available in Table 4. In most cases, the best results for each domain are obtained by training on the in-domain data. Training on all the available data helped mostly for the Koran test set. This is expected as the training data for this domain is considerably smaller than the training data for rest of the domains (Table 3). We can also see that more data is not necessarily better (Gascó et al., 2012): while the subtitles corpus is the largest of all 5 and includes 500,000 sentence pairs, it is second to last in performance as measured by the average BLEU across all test sets.

Cross-Domain BLEU vs. Cluster Proximity An interesting observation can be made with respect to the visual analysis of the domain clusters as depicted in Figure 3: as the Medical cluster (in Yellow), Law cluster (in Purple) and IT cluster (in Red) are close to each other in the embedding space, their cross-domain BLEU scores are also higher. For example, note how in the results for the Medical domain-specific model (first row in Table 4), the BLEU scores on the Law and IT test sets are much higher in comparison to those on the Koran and Subtitles test sets, which clusters are farther away in the visualized embedding space. Similarly, as the Subtitles cluster (Blue) is closer to the Koran cluster (Green), the highest cross-domain BLEU score on the Koran test set is from the Subtitles model. To further quantify this phenomenon, we plot and measure Pearson's correlation between the cosine similarity of the centroids for the English BERT-based dev sentence representations for each domain pair, and the cross-domain BLEU score for this domain pair. This is shown in Figure 4. We can see the general trend where the closer the domain centroids are (with a similarity of 1 for training and evaluating on the same domain), the higher the cross-domain BLEU is between those domains, 


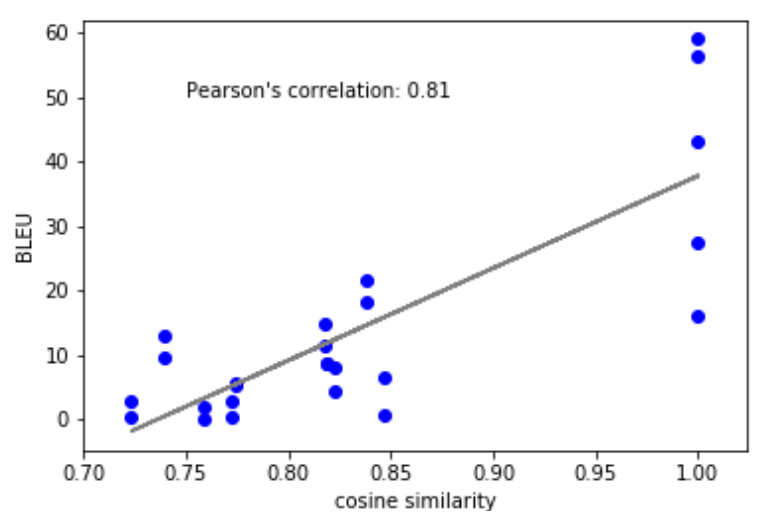

Figure 4: The cosine similarity between the centroids of the BERT representations for each domain pair vs. the corresponding cross-domain BLEU.

resulting in a Pearson's correlation of 0.81 (strong correlation). This suggests that such preliminary visual analysis can be a useful tool for understanding the relationship between diverse datasets, and motivates the use of pre-trained language model representations for domain data selection in MT.

\section{Domain Data Selection with Pretrained Language Models}

As shown in the previous section, using the right data is critical for achieving good performance on an in-domain test set, and more data is not necessarily better. However, in real-world scenarios, the availability of data labeled by domain is limited, e.g. when working with large scale, web-crawled data. In this section we focus on a data-selection scenario where only a very small number of indomain sentences are used to select data from a larger unlabeled parallel corpus. An established method for data selection was proposed by Moore and Lewis (2010), which was also used in training the winning systems in WMT 2019 (Ng et al., 2019; Barrault et al., 2019). This method compares the cross-entropy, according to domain-specific and non-domain-specific language models, for each candidate sentence for selection. The sentences are then ranked by the cross-entropy difference, and only the top sentences are selected for training.

While the method by Moore and Lewis (2010) is tried-and-true, it is based on simple n-gram language models which cannot generalize beyond the $\mathrm{n}$-grams that are seen in the in-domain set. In addition, it is restricted to the in-domain and generaldomain datasets it is trained on, which are usually small. On the contrary, pre-trained language models are trained on massive amounts of text, and, as we showed through unsupervised clustering, learn representations with domain-relevant information. In the following sections, we investigate whether this property of pretrained language models makes them useful for domain data selection.

\subsection{Methods}

We propose two methods for domain data selection with pretrained language models.

Domain-Cosine In this method we first compute a query vector, which is the element-wise average over the vector representations of the sentences in the small in-domain set. We use the same sentencelevel average-pooling approach as described in Section 2 to obtain sentence representations. We then retrieve the most relevant sentences in the training set by computing the cosine similarity of each sentence with this query vector and ranking the sentences accordingly.

Domain-Finetune It is now common knowledge that pretrained language models are especially useful when fine-tuned for the task of interest in an end-to-end manner (Ruder et al., 2019). In this method we fine-tune the pretrained LM for binary classification, where we use the in-domain sentences as positive examples, and randomly sampled general-domain sentences as negative examples. We then apply this classifier on the generaldomain data and pick the sentences that are classified as positive as in-domain, or choose the top-k sentences as ranked by the classifier output distribution. This can be seen as an instance of positiveunlabeled learning for document-set expansion; see Jacovi et al. (2019) for a recent discussion and methodology for this task.

Negative Sampling with Pre-ranking One problem that may rise when randomly sampling negative examples is that unlabeled in-domain sentences from the general-domain data may be sampled as negative examples - deteriorating the classifier performance. To alleviate this issue, we perform a biased sampling of negative examples. We first rank the general-domain data using the

\begin{tabular}{|c|c|c|c|c|c|c|}
\cline { 2 - 7 } \multicolumn{1}{c|}{} & \multicolumn{2}{c|}{ without pre-ranking } & \multicolumn{3}{c|}{ with pre-ranking } \\
\cline { 2 - 7 } \multicolumn{1}{c|}{} & $\mathrm{p}$ & $\mathrm{r}$ & $\mathrm{F} 1$ & $\mathrm{p}$ & $\mathrm{r}$ & $\mathrm{F} 1$ \\
\hline Subtitles & 0.722 & 0.984 & 0.833 & 0.964 & 0.978 & 0.971 \\
\hline Law & 0.761 & 0.94 & 0.841 & 0.944 & 0.94 & 0.942 \\
\hline Medical & 0.821 & 0.916 & 0.866 & 0.929 & 0.92 & 0.925 \\
\hline IT & 0.848 & 0.956 & 0.898 & 0.955 & 0.98 & 0.967 \\
\hline Koran & 0.966 & 0.958 & 0.962 & 0.994 & 0.974 & 0.984 \\
\hline
\end{tabular}

Table 5: Ablation analysis showing precision (p) recall (r) and F1 for the binary classification accuracy on a held-out set, with and without pre-ranking. 


\begin{tabular}{|l|c|c|c|c|c|c|}
\cline { 2 - 7 } \multicolumn{1}{c|}{} & Medical & Law & Koran & IT & Subtitles & Average \\
\hline Random-500k & 49.8 & 53.3 & 18.5 & 37.5 & 25.5 & 36.92 \\
\hline Moore-Lewis-Top-500k & 55 & 58 & 21.4 & 42.7 & 27.3 & 40.88 \\
\hline Domain-Cosine-Top-500k & 52.7 & 58 & $\mathbf{2 2}$ & 42.5 & 27.1 & 40.46 \\
\hline Domain-Finetune-Top-500k & 54.8 & 58.8 & 21.8 & $\mathbf{4 3 . 5}$ & 27.4 & $\mathbf{4 1 . 2 6}$ \\
\hline Domain-Finetune-Positive & 55.3 & 58.7 & 19.2 & 42.5 & 27 & 40.54 \\
\hline Oracle & $\mathbf{5 6 . 5}$ & $\mathbf{5 9}$ & 15.9 & 43 & 27.3 & 40.34 \\
\hline All & 53.3 & 57.2 & 20.9 & 42.1 & $\mathbf{2 7 . 6}$ & 40.22 \\
\hline
\end{tabular}

Table 6: SacreBLEU scores for the data selection experiments. Highest scores per column are marked in bold.

Domain-Cosine method, and then sample negative examples under a certain threshold in the ranking (in our experiments we sampled from the bottom two-thirds). Table 5 shows an ablation for such pre-ranking, measuring precision, recall and F1 for binary classification on a held-out set for each domain. When not using pre-ranking, as the training data for the domain is larger, the precision is lower - since more in-domain examples are drawn as negative samples. Using pre-ranking indeed alleviates this issue, achieving higher F1 scores in all cases. Given the results in Table 5 we always use pre-ranking in the following experiments.

\subsection{Experimental Setup}

We perform data selection experiments for each domain in the multi-domain dataset. As the small set of monolingual in-domain data we take the 2000 development sentences from each domain. For the general-domain corpus we concatenate the training data from all domains, resulting in 1,456,317 sentences. To enable faster experimentation we used DistilBERT (Sanh et al., 2019) for the DomainCosine and Domain-Finetune methods. More technical details are available in the supplementary material. We compare our methods to four approaches: (1) The established method by Moore and Lewis (2010), (2) a random selection baseline, (3) an oracle which is trained on all the available in-domain data, and (4) the model we train on all the domains concatenated. We select the top 500k examples to cover the size of every specific in-domain dataset. We train Transformer NMT models on the selected data with a similar configuration to the ones trained in the cross-domain evaluation.

\subsection{Results}

The results are available in Table 6 . We can see that all selection methods performed much better in terms of BLEU than random selection. It is also nice to see that all selection methods performed better than using all the available data or the oracle-selected data when averaged across all

\begin{tabular}{|c|c|c|c|c|c|c|}
\cline { 2 - 7 } \multicolumn{1}{c|}{} & \multicolumn{2}{c|}{ Moore-Lewis } & \multicolumn{2}{c|}{ D-Cosine } & \multicolumn{2}{c|}{ D-Finetune } \\
\cline { 2 - 7 } \multicolumn{1}{c|}{} & $\mathrm{p}$ & $\mathrm{r}$ & $\mathrm{p}$ & $\mathrm{r}$ & $\mathrm{p}$ & $\mathrm{r}$ \\
\hline Medical & 0.476 & 0.955 & 0.391 & 0.788 & 0.485 & 0.975 \\
\hline Law & 0.836 & 0.894 & 0.841 & 0.899 & 0.902 & 0.965 \\
\hline Koran & 0.35 & 0.985 & 0.36 & 0.989 & 0.36 & 0.998 \\
\hline IT & 0.441 & 0.985 & 0.382 & 0.857 & 0.447 & 0.998 \\
\hline Subtitles & 0.899 & 0.899 & 0.916 & 0.916 & 0.957 & 0.957 \\
\hline Average & 0.6 & 0.944 & 0.578 & 0.89 & 0.63 & 0.979 \\
\hline
\end{tabular}

Table 7: Precision (p) and recall (r) for data selection of $500 \mathrm{k}$ sentences with respect to the oracle selection.

domains, showing again that more data is not necessarily better in multi-domain scenarios and that data selection is a useful approach. Regarding a comparison of the data selection methods, MooreLewis performed better than Domain-Cosine, while Domain-Finetune performed best, showing the benefit of fine-tuning large pretrained models for the data selection task. Using the positively-labeled examples alone (Domain-Finetune-Positive) performed worse than using the top 500k examples but better than Domain-Cosine, while not requiring to determine the number of selected sentences.

\subsection{Analysis}

We perform an analysis on the selected datasets, where we measure the precision and recall of sentence selection with respect to the oracle selection. The results are available in Table 7. As also reflected in the BLEU scores, the Domain-Finetune method resulted in the highest domain recall with a minimum of 97.5, while Moore-Lewis and DomainCosine scored 89.4 and 78.8 respectively. We find these results very appealing given that only 2000 in-domain sentences were used for selection for each domain out of 1.45 million sentences. Also note that we used DistilBERT in these experiments: we believe that using larger, non-distilled models may result in even better selection performance (although at the price of larger computational requirements).

\section{Related Work}

Previous works used n-gram LMs for data selection (Moore and Lewis, 2010; Axelrod et al., 2011) or 
other count-based methods (Axelrod, 2017; Poncelas et al., 2018; Parcheta et al., 2018; Santamaría and Axelrod, 2019). While such methods work well in practice, they cannot generalize beyond the $\mathrm{N}$-grams observed in the in-domain datasets, which are usually small.

Duh et al. (2013) proposed to replace n-gram models with RNN-based LMs with notable improvements. However, such methods do not capture the rich sentence-level global context as in the recent self-attention-based MLMs; as we showed in the clustering experiments, autoregressive neural LMs were inferior to masked LMs in clustering the data by domain. In addition, training large LMs may be prohibitive without relying on pre-training.

Regarding domain clustering for MT, Hasler et al. (2014) discovered topics using LDA instead of using domain labels. Cuong et al. (2016) induced latent subdomains from the training data using a dedicated probabilistic model.

Many works used vector-based retrieval for data selection; Ruder and Plank (2017) learn to select data using Bayesian optimization, and explored word2vec for that purpose. Duma and Menzel (2016) create paragraph vectors for data selection in the context of SMT. Wang et al. (2017) use internal representations from the NMT model to perform data selection. Bapna and Firat (2019) propose a mechanism for incorporating retrieved sentences for each instance for domain adaptation in NMT, using representations extracted from a pretrained NMT model. Farajian et al. (2017) explored instance-based data selection in a multi-domain scenario using information retrieval methods.

Other related works on domain adaptation include Dou et al. (2019a) that adapts multi-domain NMT models with domain-aware feature embeddings, which are learned via an auxiliary language modeling task. Peris et al. (2017) proposed neuralnetwork based classifiers for data selection in SMT. For more related work on data selection and domain adaptation in the context of MT, see the surveys by Eetemadi et al. (2015) for SMT and more recently Chu and Wang (2018) for NMT.

Unrelated to MT, Ma et al. (2019) used BERT to select data for tasks from the GLUE benchmark (Wang et al., 2018). However, they assumed supervision for all the different tasks/domains, while we propose an unsupervised method requiring only a small set of in-domain data. Also in the context of pretrained language models, Gururangan et al. (2020) show the importance of additional pretraining with in-domain data to improve the downstream task-specific performance.

While previous work made important contributions to domain data selection, our work is the first to explore massive pretrained language models for both unsupervised domain clustering and for data selection in NMT.

\section{Conclusions and Future Work}

We showed that massive pre-trained language models are highly effective in mapping data to domains in a fully-unsupervised manner using averagepooled sentence representations and GMM-based clustering. We suggest that such clusters are a more appropriate, data driven approach to domains in natural language than simplistic labels (e.g. "medical text"), and that it will improve over time as better and larger pretrained LMs will become available. We proposed new methods to harness this property for domain data selection using distance-based ranking in vector space and pretrained LM finetuning, requiring only a small set of in-domain data. We demonstrated the effectiveness of our methods on a new, improved data split we created for a previously studied multi-domain machine translation benchmark. Our methods perform similarly or better than an established data selection method and oracle in-domain training across all five domains in the benchmark.

This work just scratches the surface with what can be done on the subject; possible avenues for future work include extending this with multilingual data selection and multilingual LMs (Conneau and Lample, 2019; Conneau et al., 2019; Wu et al., 2019; Hu et al., 2020), using such selection methods with domain-curriculum training (Zhang et al., 2019; Wang et al., 2019b), applying them on noisy, web-crawled data (Junczys-Dowmunt, 2018) or for additional tasks (Gururangan et al., 2020). Another interesting avenue is applying this to unsupervised NMT, which is highly sensitive to domain mismatch (Marchisio et al., 2020; Kim et al., 2020). We hope this work will encourage more research on finding the right data for the task, towards more efficient and robust NLP.

\section{Acknowledgements}

We thank Wei Wang for early discussions on domain adaptation and data selection that inspired this work during Roee's internship in Google Translate. 


\section{References}

Roee Aharoni and Yoav Goldberg. 2018. Split and rephrase: Better evaluation and stronger baselines. In Proceedings of the 56th Annual Meeting of the Association for Computational Linguistics (Volume 2: Short Papers), pages 719-724, Melbourne, Australia. Association for Computational Linguistics.

Mikko Aulamo and Jörg Tiedemann. 2019. The OPUS resource repository: An open package for creating parallel corpora and machine translation services. In Proceedings of the 22nd Nordic Conference on Computational Linguistics, pages 389-394, Turku, Finland. Linköping University Electronic Press.

Amittai Axelrod. 2017. Cynical selection of language model training data. arXiv preprint arXiv:1709.02279.

Amittai Axelrod, Xiaodong He, and Jianfeng Gao. 2011. Domain adaptation via pseudo in-domain data selection. In Proceedings of the 2011 Conference on Empirical Methods in Natural Language Processing, pages 355-362, Edinburgh, Scotland, UK. Association for Computational Linguistics.

Ankur Bapna and Orhan Firat. 2019. Non-parametric adaptation for neural machine translation. In Proceedings of the 2019 Conference of the North American Chapter of the Association for Computational Linguistics: Human Language Technologies, Volume 1 (Long and Short Papers), pages 1921-1931, Minneapolis, Minnesota. Association for Computational Linguistics.

Loïc Barrault, Ondřej Bojar, Marta R. Costa-jussà, Christian Federmann, Mark Fishel, Yvette Graham, Barry Haddow, Matthias Huck, Philipp Koehn, Shervin Malmasi, Christof Monz, Mathias Müller, Santanu Pal, Matt Post, and Marcos Zampieri. 2019. Findings of the 2019 conference on machine translation (WMT19). In Proceedings of the Fourth Conference on Machine Translation (Volume 2: Shared Task Papers, Day 1), pages 1-61, Florence, Italy. Association for Computational Linguistics.

David M Blei, Andrew Y Ng, and Michael I Jordan. 2003. Latent dirichlet allocation. Journal of machine Learning research, 3(Jan):993-1022.

Chenhui Chu and Rui Wang. 2018. A survey of domain adaptation for neural machine translation. In Proceedings of the 27th International Conference on Computational Linguistics, pages 1304-1319, Santa Fe, New Mexico, USA. Association for Computational Linguistics.

Kevin Clark, Urvashi Khandelwal, Omer Levy, and Christopher D Manning. 2019. What does BERT look at? an analysis of BERT's attention. arXiv preprint arXiv:1906.04341.

Alexis Conneau, Kartikay Khandelwal, Naman Goyal, Vishrav Chaudhary, Guillaume Wenzek, Francisco
Guzmán, Edouard Grave, Myle Ott, Luke Zettlemoyer, and Veselin Stoyanov. 2019. Unsupervised cross-lingual representation learning at scale. arXiv preprint arXiv:1911.02116.

Alexis Conneau and Guillaume Lample. 2019. Crosslingual language model pretraining. In Advances in Neural Information Processing Systems, pages 7057-7067.

Susan M Conrad and Douglas Biber. 2005. The frequency and use of lexical bundles in conversation and academic prose. Lexicographica.

Hoang Cuong, Khalil Sima'an, and Ivan Titov. 2016. Adapting to all domains at once: Rewarding domain invariance in SMT. Transactions of the Association for Computational Linguistics, 4:99-112.

Hal Daume. 2009. K-means vs GMM, sum-product vs max-product.

Jacob Devlin, Ming-Wei Chang, Kenton Lee, and Kristina Toutanova. 2019. BERT: Pre-training of deep bidirectional transformers for language understanding. In Proceedings of the 2019 Conference of the North American Chapter of the Association for Computational Linguistics: Human Language Technologies, Volume 1 (Long and Short Papers), pages 4171-4186, Minneapolis, Minnesota. Association for Computational Linguistics.

Zi-Yi Dou, Junjie Hu, Antonios Anastasopoulos, and Graham Neubig. 2019a. Unsupervised domain adaptation for neural machine translation with domainaware feature embeddings. In Proceedings of the 2019 Conference on Empirical Methods in Natural Language Processing and the 9th International Joint Conference on Natural Language Processing (EMNLP-IJCNLP), pages 1417-1422, Hong Kong, China. Association for Computational Linguistics.

Zi-Yi Dou, Xinyi Wang, Junjie Hu, and Graham Neubig. 2019b. Domain differential adaptation for neural machine translation. In Proceedings of the $3 \mathrm{rd}$ Workshop on Neural Generation and Translation, Hong Kong. Association for Computational Linguistics.

Kevin Duh, Graham Neubig, Katsuhito Sudoh, and Hajime Tsukada. 2013. Adaptation data selection using neural language models: Experiments in machine translation. In Proceedings of the 51st Annual Meeting of the Association for Computational Linguistics (Volume 2: Short Papers), pages 678-683, Sofia, Bulgaria. Association for Computational Linguistics.

Mirela-Stefania Duma and Wolfgang Menzel. 2016. Data selection for IT texts using paragraph vector. In Proceedings of the First Conference on Machine Translation: Volume 2, Shared Task Papers, pages 428-434, Berlin, Germany. Association for Computational Linguistics. 
Sauleh Eetemadi, William Lewis, Kristina Toutanova, and Hayder Radha. 2015. Survey of data-selection methods in statistical machine translation. Machine Translation, 29(3-4):189-223.

M. Amin Farajian, Marco Turchi, Matteo Negri, and Marcello Federico. 2017. Multi-domain neural machine translation through unsupervised adaptation. In Proceedings of the Second Conference on Machine Translation, pages 127-137, Copenhagen, Denmark. Association for Computational Linguistics.

Guillem Gascó, Martha-Alicia Rocha, Germán Sanchis-Trilles, Jesús Andrés-Ferrer, and Francisco Casacuberta. 2012. Does more data always yield better translations? In Proceedings of the 13th Conference of the European Chapter of the Association for Computational Linguistics, pages 152-161, Avignon, France. Association for Computational Linguistics.

Yoav Goldberg. 2019. Assessing BERT's syntactic abilities. arXiv preprint arXiv:1901.05287.

Suchin Gururangan, Ana Marasovi, Swabha Swayamdipta, Kyle Lo, Iz Beltagy, Doug Downey, and Noah A. Smith. 2020. Don't stop pretraining: Adapt language models to domains and tasks. ACL.

Gholamreza Haffari, Maxim Roy, and Anoop Sarkar. 2009. Active learning for statistical phrase-based machine translation. In Proceedings of Human Language Technologies: The 2009 Annual Conference of the North American Chapter of the Association for Computational Linguistics, pages 415-423, Boulder, Colorado. Association for Computational Linguistics.

Eva Hasler, Phil Blunsom, Philipp Koehn, and Barry Haddow. 2014. Dynamic topic adaptation for phrase-based MT. In Proceedings of the 14th Conference of the European Chapter of the Association for Computational Linguistics, pages 328337, Gothenburg, Sweden. Association for Computational Linguistics.

Kenneth Heafield. 2011. KenLM: Faster and smaller language model queries. In Proceedings of the Sixth Workshop on Statistical Machine Translation, pages 187-197, Edinburgh, Scotland. Association for Computational Linguistics.

Junjie Hu, Sebastian Ruder, Aditya Siddhant, Graham Neubig, Orhan Firat, and Melvin Johnson. 2020. Xtreme: A massively multilingual multi-task benchmark for evaluating cross-lingual generalization. arXiv preprint arXiv:2003.11080.

Junjie Hu, Mengzhou Xia, Graham Neubig, and Jaime Carbonell. 2019. Domain adaptation of neural machine translation by lexicon induction. In Proceedings of the 57th Annual Meeting of the Association for Computational Linguistics, Florence, Italy. Association for Computational Linguistics.
Alon Jacovi, Gang Niu, Yoav Goldberg, and Masashi Sugiyama. 2019. Scalable evaluation and improvement of document set expansion via neural positive-unlabeled learning. arXiv preprint arXiv:1910.13339.

Marcin Junczys-Dowmunt. 2018. Dual conditional cross-entropy filtering of noisy parallel corpora. In Proceedings of the Third Conference on Machine Translation: Shared Task Papers, pages 888-895, Belgium, Brussels. Association for Computational Linguistics.

Yunsu Kim, Miguel Graça, and Hermann Ney. 2020. When and why is unsupervised neural machine translation useless? arXiv preprint arXiv:2004.10581.

Diederik P Kingma and Jimmy Ba. 2014. Adam: A method for stochastic optimization. arXiv preprint arXiv:1412.6980.

Philipp Koehn, Hieu Hoang, Alexandra Birch, Chris Callison-Burch, Marcello Federico, Nicola Bertoldi, Brooke Cowan, Wade Shen, Christine Moran, Richard Zens, Chris Dyer, Ondřej Bojar, Alexandra Constantin, and Evan Herbst. 2007. Moses: Open source toolkit for statistical machine translation. In Proceedings of the 45th Annual Meeting of the Association for Computational Linguistics Companion Volume Proceedings of the Demo and Poster Sessions, pages 177-180, Prague, Czech Republic. Association for Computational Linguistics.

Philipp Koehn and Rebecca Knowles. 2017. Six challenges for neural machine translation. In Proceedings of the First Workshop on Neural Machine Translation, pages 28-39, Vancouver. Association for Computational Linguistics.

Katherine Lee, Orhan Firat, Ashish Agarwal, Clara Fannjiang, and David Sussillo. 2018. Hallucinations in neural machine translation.

Yinhan Liu, Myle Ott, Naman Goyal, Jingfei Du, Mandar Joshi, Danqi Chen, Omer Levy, Mike Lewis, Luke Zettlemoyer, and Veselin Stoyanov. 2019. Roberta: A robustly optimized bert pretraining approach. arXiv preprint arXiv:1907.11692.

Xiaofei Ma, Peng Xu, Zhiguo Wang, Ramesh Nallapati, and Bing Xiang. 2019. Domain adaptation with BERT-based domain classification and data selection. In Proceedings of the 2nd Workshop on Deep Learning Approaches for Low-Resource NLP (DeepLo 2019), pages 76-83, Hong Kong, China. Association for Computational Linguistics.

Christopher D Manning, Prabhakar Raghavan, and Hinrich Schütze. 2008. Introduction to information retrieval. Cambridge university press.

Kelly Marchisio, Kevin Duh, and Philipp Koehn. 2020. When does unsupervised machine translation work? arXiv preprint arXiv:2004.05516. 
Tomas Mikolov, Ilya Sutskever, Kai Chen, Greg S Corrado, and Jeff Dean. 2013. Distributed representations of words and phrases and their compositionality. In Advances in neural information processing systems, pages 3111-3119.

Robert C. Moore and William Lewis. 2010. Intelligent selection of language model training data. In Proceedings of the ACL 2010 Conference Short Papers, pages 220-224, Uppsala, Sweden. Association for Computational Linguistics.

Mathias Müller, Annette Rios, and Rico Sennrich. 2019. Domain robustness in neural machine translation.

Nathan Ng, Kyra Yee, Alexei Baevski, Myle Ott, Michael Auli, and Sergey Edunov. 2019. Facebook FAIR's WMT19 news translation task submission. In Proceedings of the Fourth Conference on Machine Translation (Volume 2: Shared Task Papers, Day 1), pages 314-319, Florence, Italy. Association for Computational Linguistics.

Xing Niu, Marianna Martindale, and Marine Carpuat. 2017. A study of style in machine translation: Controlling the formality of machine translation output. In Proceedings of the 2017 Conference on Empirical Methods in Natural Language Processing, pages 2814-2819, Copenhagen, Denmark. Association for Computational Linguistics.

Myle Ott, Sergey Edunov, Alexei Baevski, Angela Fan, Sam Gross, Nathan Ng, David Grangier, and Michael Auli. 2019. fairseq: A fast, extensible toolkit for sequence modeling. In Proceedings of the 2019 Conference of the North American Chapter of the Association for Computational Linguistics (Demonstrations), pages 48-53, Minneapolis, Minnesota. Association for Computational Linguistics.

Zuzanna Parcheta, Germán Sanchis-Trilles, and Francisco Casacuberta. 2018. Data selection for NMT using infrequent n-gram recovery. EAMT 2018.

F. Pedregosa, G. Varoquaux, A. Gramfort, V. Michel, B. Thirion, O. Grisel, M. Blondel, P. Prettenhofer, R. Weiss, V. Dubourg, J. Vanderplas, A. Passos, D. Cournapeau, M. Brucher, M. Perrot, and E. Duchesnay. 2011. Scikit-learn: Machine learning in Python. Journal of Machine Learning Research, 12:2825-2830.

Álvaro Peris, Mara Chinea-Ríos, and Francisco Casacuberta. 2017. Neural networks classifier for data selection in statistical machine translation. The Prague Bulletin of Mathematical Linguistics, 108(1):283-294.

Matthew Peters, Mark Neumann, Mohit Iyyer, Matt Gardner, Christopher Clark, Kenton Lee, and Luke Zettlemoyer. 2018. Deep contextualized word representations. In Proceedings of the 2018 Conference of the North American Chapter of the Association for Computational Linguistics: Human Language Technologies, Volume 1 (Long Papers), pages
2227-2237, New Orleans, Louisiana. Association for Computational Linguistics.

Alberto Poncelas, Gideon Maillette de Buy Wenniger, and Andy Way. 2018. Data selection with feature decay algorithms using an approximated target side. arXiv preprint arXiv:1811.03039.

Matt Post. 2018. A call for clarity in reporting BLEU scores. In Proceedings of the Third Conference on Machine Translation: Research Papers, pages 186191, Belgium, Brussels. Association for Computational Linguistics.

Ofir Press and Lior Wolf. 2017. Using the output embedding to improve language models. In Proceedings of the 15th Conference of the European Chapter of the Association for Computational Linguistics. Volume 2, Short Papers, pages 157-163, Valencia, Spain. Association for Computational Linguistics.

Alec Radford, Karthik Narasimhan, Tim Salimans, and Ilya Sutskever. 2018. Improving language understanding by generative pre-training. OpenAI blog.

Colin Raffel, Noam Shazeer, Adam Roberts, Katherine Lee, Sharan Narang, Michael Matena, Yanqi Zhou, Wei Li, and Peter J. Liu. 2019. Exploring the limits of transfer learning with a unified text-to-text transformer.

Sebastian Ruder, Matthew E. Peters, Swabha Swayamdipta, and Thomas Wolf. 2019. Transfer learning in natural language processing. In Proceedings of the 2019 Conference of the North American Chapter of the Association for Computational Linguistics: Tutorials, pages 15-18, Minneapolis, Minnesota. Association for Computational Linguistics.

Sebastian Ruder and Barbara Plank. 2017. Learning to select data for transfer learning with Bayesian optimization. In Proceedings of the 2017 Conference on Empirical Methods in Natural Language Processing, pages 372-382, Copenhagen, Denmark. Association for Computational Linguistics.

Hassan Sajjad, Nadir Durrani, Fahim Dalvi, Yonatan Belinkov, and Stephan Vogel. 2017. Neural machine translation training in a multi-domain scenario. arXiv preprint arXiv:1708.08712.

Victor Sanh, Lysandre Debut, Julien Chaumond, and Thomas Wolf. 2019. Distilbert, a distilled version of BERT: smaller, faster, cheaper and lighter. arXiv preprint arXiv:1910.01108.

Lucía Santamaría and Amittai Axelrod. 2019. Data selection with cluster-based language difference models and cynical selection. arXiv preprint arXiv:1904.04900.

Rico Sennrich, Barry Haddow, and Alexandra Birch. 2016. Neural machine translation of rare words with subword units. In Proceedings of the 54th Annual Meeting of the Association for Computational 
Linguistics (Volume 1: Long Papers), pages 17151725, Berlin, Germany. Association for Computational Linguistics.

Catarina Cruz Silva, Chao-Hong Liu, Alberto Poncelas, and Andy Way. 2018. Extracting in-domain training corpora for neural machine translation using data selection methods. In Proceedings of the Third Conference on Machine Translation: Research Papers, pages 224-231, Belgium, Brussels. Association for Computational Linguistics.

Ian Tenney, Dipanjan Das, and Ellie Pavlick. 2019. BERT rediscovers the classical NLP pipeline. In Proceedings of the 57th Annual Meeting of the Association for Computational Linguistics, pages 4593 4601, Florence, Italy. Association for Computational Linguistics.

Jörg Tiedemann. 2012. Parallel data, tools and interfaces in OPUS. In Proceedings of the Eighth International Conference on Language Resources and Evaluation (LREC-2012), pages 2214-2218, Istanbul, Turkey. European Languages Resources Association (ELRA).

Ashish Vaswani, Noam Shazeer, Niki Parmar, Jakob Uszkoreit, Llion Jones, Aidan N Gomez, Ł ukasz Kaiser, and Illia Polosukhin. 2017. Attention is all you need. In I. Guyon, U. V. Luxburg, S. Bengio, H. Wallach, R. Fergus, S. Vishwanathan, and R. Garnett, editors, Advances in Neural Information Processing Systems 30, pages 5998-6008. Curran Associates, Inc.

Alex Wang, Yada Pruksachatkun, Nikita Nangia, Amanpreet Singh, Julian Michael, Felix Hill, Omer Levy, and Samuel R Bowman. 2019a. Superglue: A stickier benchmark for general-purpose language understanding systems. arXiv preprint arXiv:1905.00537.

Alex Wang, Amanpreet Singh, Julian Michael, Felix Hill, Omer Levy, and Samuel Bowman. 2018. GLUE: A multi-task benchmark and analysis platform for natural language understanding. In Proceedings of the 2018 EMNLP Workshop BlackboxNLP: Analyzing and Interpreting Neural Networks for NLP, pages 353-355, Brussels, Belgium. Association for Computational Linguistics.

Rui Wang, Andrew Finch, Masao Utiyama, and Eiichiro Sumita. 2017. Sentence embedding for neural machine translation domain adaptation. In Proceedings of the 55th Annual Meeting of the Association for Computational Linguistics (Volume 2: Short Papers), pages 560-566, Vancouver, Canada. Association for Computational Linguistics.

Wei Wang, Isaac Caswell, and Ciprian Chelba. 2019b. Dynamically composing domain-data selection with clean-data selection by "co-curricular learning" for neural machine translation. In Proceedings of the 57th Annual Meeting of the Association for Computational Linguistics, pages 1282-1292, Florence, Italy. Association for Computational Linguistics.
Marlies van der Wees. 2017. What's in a Domain? Towards Fine-Grained Adaptation for Machine Translation. Ph.D. thesis, University of Amsterdam.

Marlies van der Wees, Arianna Bisazza, Wouter Weerkamp, and Christof Monz. 2015. What's in a domain? analyzing genre and topic differences in statistical machine translation. In Proceedings of the 53rd Annual Meeting of the Association for Computational Linguistics and the 7th International Joint Conference on Natural Language Processing (Volume 2: Short Papers), pages 560-566, Beijing, China. Association for Computational Linguistics.

Thomas Wolf, Lysandre Debut, Victor Sanh, Julien Chaumond, Clement Delangue, Anthony Moi, Pierric Cistac, Tim Rault, R'emi Louf, Morgan Funtowicz, and Jamie Brew. 2019. Huggingface's transformers: State-of-the-art natural language processing. ArXiv, abs/1910.03771.

Shijie Wu, Alexis Conneau, Haoran Li, Luke Zettlemoyer, and Veselin Stoyanov. 2019. Emerging cross-lingual structure in pretrained language models. arXiv preprint arXiv:1911.01464.

Zhilin Yang, Zihang Dai, Yiming Yang, Jaime Carbonell, Ruslan Salakhutdinov, and Quoc V Le. 2019. XLNet: Generalized autoregressive pretraining for language understanding. arXiv preprint arXiv:1906.08237.

Xuan Zhang, Pamela Shapiro, Gaurav Kumar, Paul McNamee, Marine Carpuat, and Kevin Duh. 2019. Curriculum learning for domain adaptation in neural machine translation. In Proceedings of the 2019 Conference of the North American Chapter of the Association for Computational Linguistics: Human Language Technologies, Volume 1 (Long and Short Papers), pages 1903-1915, Minneapolis, Minnesota. Association for Computational Linguistics. 


\section{A Appendix}

\section{A.1 NMT Training}

Figure 5 details the hyperparameter configuration we used to train the NMT models. We use Transformer models (Vaswani et al., 2017) in the Base configuration using the implementation provided in Fairseq (Ott et al., 2019). For all models we use a joint BPE vocabulary (Sennrich et al., 2016) learned with $32 \mathrm{k}$ merge operations over the concatenated corpus in both languages, enabling to tie all the embedding layers (Press and Wolf, 2017). ${ }^{12}$ We perform early stopping if the BLEU score on the domain-specific development set did not improve in 10 consequent checkpoints. We use the ADAM (Kingma and Ba, 2014) optimizer with an initial learning rate of $5 \cdot 10^{-} 4$ and a maximum of 4096 tokens per batch. We trained all models on a single NVIDIA GPU. We decode using beam search with a beam size of 5. For pre-processing we used the Moses (Koehn et al., 2007) pipeline including tokenization, normalize-punctuation, nonprinting character removal, truecasing and cleaning. We removed examples with sequences longer than 100 tokens from the training data (before subword segmentation).

\section{A.2 Data Split}

Table 8 shows details about the overlap between the training, development and test sets for the different data splits of the multi-domain dataset. The overlap was computed using the English part of the corpus.

\section{A.3 GMM Clustering}

We learn GMMs with full covariance matrices, i.e. without constraints on covariance matrices that determine the shape of each component in the mixture, as implemented in scikit-learn (Pedregosa et al., 2011). We train the models until convergence or for a maximum of $150 \mathrm{EM}$ iterations.

\section{A.4 Language Model Finetuning}

We fine-tune the binary classification head for 5 epochs. We use the ADAM (Kingma and Ba, 2014) optimizer with an initial learning rate of $2 \cdot 10^{-} 5$. We train the model using 4 NVIDIA GPUs with 256 sentences per batch (64 per GPU).

\footnotetext{
${ }^{12}$ We used the implementation in https://github. com/rsennrich/subword-nmt
}

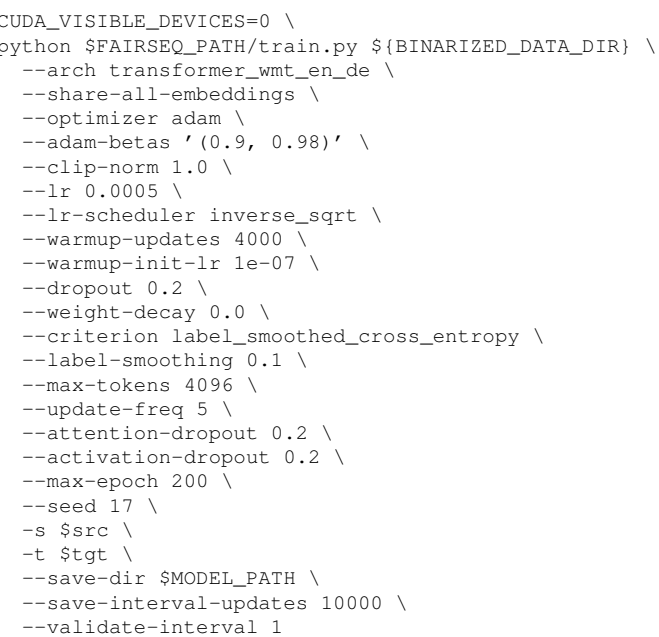

Figure 5: The hyperparameter configuration we used for NMT model training using Fairseq (Ott et al., 2019).

\section{A.5 Moore-Lewis Implementation}

We used the implementation of Moore and Lewis (2010) by Pamela Shapiro, as available in: https://github.com/pamelashapiro/ moore-lewis. This implementation uses the KenLM N-Gram language model toolkit (Heafield, 2011).

\section{A.6 Additional Visualizations}

Figure 6 shows visualizations of the multi-domain dataset from additional pre-trained masked language models (BERT large and RoBERTa), and Figure 7 shows the same visualization for autoregressive models (XLNet and GPT2). 


\begin{tabular}{|c|c|c|c|c|}
\cline { 3 - 5 } \multicolumn{2}{c|}{} & Koehn and Knowles (2017) & Müller et al. (2019) & New Split \\
\hline \multirow{4}{*}{$\begin{array}{c}* \\
\text { in dev } \text { drain }\end{array}$} & Medical & $1090 / 2000(54.5 \%)$ & $1204 / 2000(60.2 \%)$ & $0 / 2000$ \\
\cline { 2 - 5 } & Koran & $0 / 2000$ & $1926 / 2000(96.3)$ & $0 / 2000$ \\
\cline { 2 - 5 } & Subtitles & $1183 / 5000(23.66 \%)$ & $638 / 2000(31.9 \%)$ & $0 / 2000$ \\
\cline { 2 - 5 } & Law & $595 / 2000(29.75 \%)$ & $1000 / 2000(50 \%)$ & $0 / 2000$ \\
\cline { 2 - 5 } & IT & $2496 / 2526(98.81 \%)$ & $783 / 2000(39.15 \%)$ & $0 / 2000$ \\
\hline \multirow{4}{*}{$\begin{array}{c}\% \text { test } \\
\text { in train }\end{array}$} & Medical & $571 / 2000(28.55 \%)$ & $516 / 1691(30.51 \%)$ & $0 / 2000$ \\
\cline { 2 - 5 } & Koran & $0 / 2000$ & $1949 / 2000(97.45 \%)$ & $0 / 2000$ \\
\cline { 2 - 5 } & Subtitles & $451 / 5000(9.02 \%)$ & $478 / 2000(23.9 \%)$ & $0 / 2000$ \\
\cline { 2 - 5 } & Law & $649 / 2000(32.45 \%)$ & $966 / 2000(48.3 \%)$ & $0 / 2000$ \\
\cline { 2 - 5 } & IT & $945 / 1856(50.92 \%)$ & $1036 / 2000(51.8 \%)$ & $0 / 2000$ \\
\hline
\end{tabular}

Table 8: Details about the different data splits for the multi-domain corpus. 

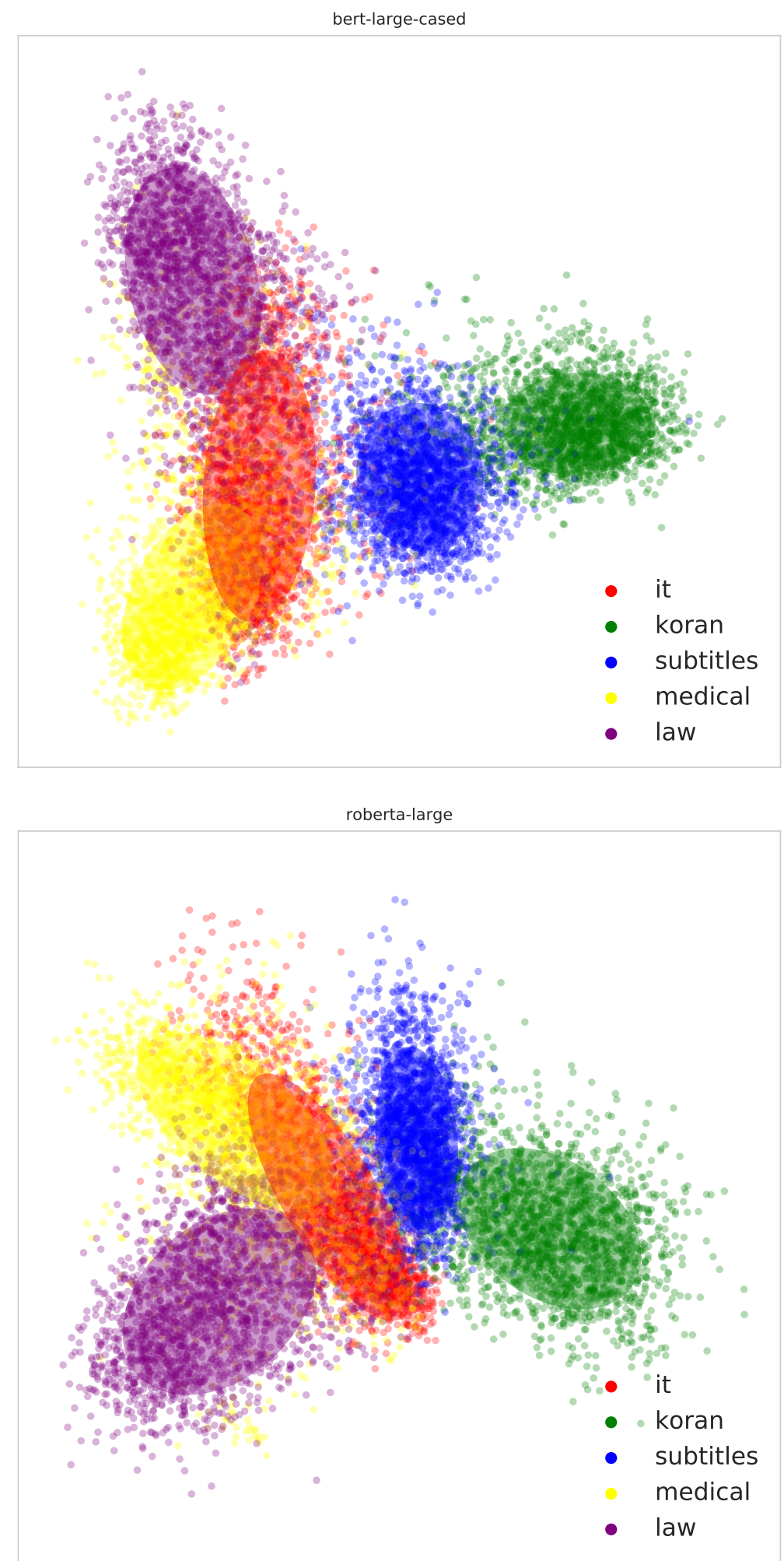

Figure 6: 2D visualizations of the unsupervised GMM-based clustering for different pretrained MLMs. 

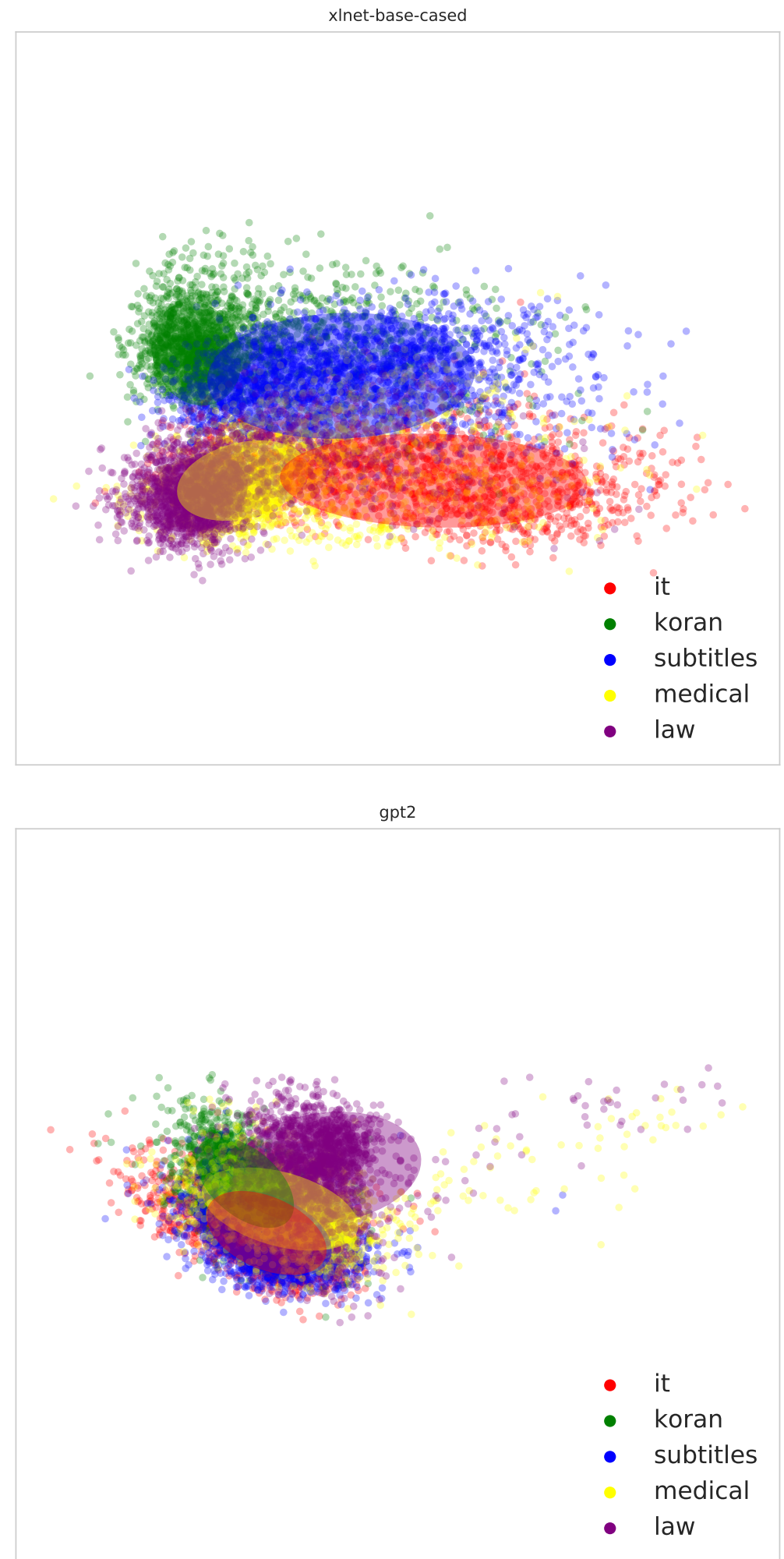

Figure 7: 2D visualizations of the unsupervised GMM-based clustering for different pretrained auto-regressive LMs. 